\title{
VASO-OCCLUSIVE RETINOPATHY AND STAGE IV LUPUS NEPHRITIS; PRIMARY MANIFESTATION OF A 30 YEAR OLD FEMALE
}

\author{
TASNUVA SAIFUL ${ }^{1}$, QUADRAT-E- ELAHI ${ }^{2}$, MAMUN MOSTAFI $^{3}$ ABDULALI MIA ${ }^{4}$
}

\begin{abstract}
The diagnosis of systemic lupus erythematosus (SLE) depends on clinical evidence of renal, rheumatologic, cutaneous, and neurologic involvement, supported by serological markers. Ocular involvement is not included in diagnostic criteria of SLE. Vaso-occlusive retinopathy, as the primary manifestation of systemic lupus erythematosus (SLE), is relatively rare. We report a 30-year-old female, who suddenly suffered bilateral visual loss. Fundus examination revealed bilateral occlusive retinal vasculitis. She fulfilled the American College of Rheumatology criteria for diagnosing SLE; she had arthralgia, photosensitivity, positive ANA, high titre of anti-dsDNA, and proteinuria. A renal biopsy showed stage IV lupus nephritis. Treatment was initiated with pulse steroid and immunosuppressant. Best corrected visual acuity of both eye improved to finger counting after treatment. This case demonstrates that in patients with severe vaso-occlusive retinopathy, a generalized immunological disorder, like SLE, should be suspected.
\end{abstract}

Keywords: occlusive retinal vasculitis, systemic lupus erythematosus, lupus nephrtis.

Received: 02 March 2017

Accepted: 28 November 2017

DOI: http:// dx.doi.org/10.3329/bjmed.v29i1.35406

\section{Introduction:}

The first use of the term "lupus," which is Latin for wolf, is attributed to the 12 th-century physician Rogerius to describe the classic malar rash that resembles the pattern or fur on a wolf's face. Systemic lupus erythematosus (SLE) is a chronic systemic autoimmune disease characterized by the production and deposition of autoantibody immune complexes in tissues throughout the body ${ }^{1}$. Systemic lupus erythematosus can affect virtually any organ in the body, and the American College of Rheumatology has set criteria for the diagnosis of SLE. Although ocular manifestations are not part of the diagnostic criteria, they are common and can be observed in up to onethird of patients with $\mathrm{SLE}^{2}$. The most common ophthalmic presentation in patients with SLE is retinopathy with microangiopathy, which presents with cotton wool spots with or without intraretinal hemorrhage ${ }^{3}$. However, severe vaso-occlusive retinopathy as the initial manifestation of SLE is relatively rare ${ }^{4}$. There have been fewer than 50 patients previously reported in the literature (4-8). Approximately $35 \%$ of adults with SLE have clinical evidence of nephritis at the time of diagnosis, with an estimated total of 50-60\% developing nephritis during the first 10 years of disease ${ }^{(9-12)}$. We, herein, describe a case of systemic lupus erythematosus, which who initially presented with bilateral vasoocclusive retinopathy.

\section{Case report:}

A 30 years old female was presented with sudden onset of visual loss for 03 days with a recent history of high grade intermittent fever and lower limb swelling for 12 days. She had no history of headache, convulsion, vomiting, diarrhea, trauma, documented hypoglycemia, recent change of medication or recent travel.

On details query, she gave history of photosensitivity, arthralgia and alopecia for last 2-3 months. She used

1. Classified medicine specialist, $\mathrm{CMH}$, Dhaka

2. Classified medicine specialists, $\mathrm{CMH}$, Dhaka

3. Chief Physicians, CMH, Dhaka

4. Consultant Physician General, Bangladesh Armed Forces

Address of Correspondence: Dr. Tasnuva Saiful, Classified medicine Specialist, CMH, Dhaka

Bangladesh J Medicine 2018; $29: 36-40$ 
NSAIDS sometimes for joint pain but denied having any skin lesion. On admission, her body temperature was $104^{\circ} \mathrm{F}$, heart rate was $104 /$ minute, respiratory rate was $18 /$ minute, and blood pressure was $110 / 60$ $\mathrm{mm} \mathrm{Hg}$. Anaemic conjunctiva was found, bilateral pedal edema was present. She was referred to an ophthalmologist for visual loss. By using semi quantitative scale her visual acuity (VA) was limited to light perception. Retinal examination revealed multiple areas of hemorrhage and exudate involving macula. She was advised for fluorescence angiogram. Color photograph of the fundus of both eyes showed dilatation, tortuosity and beading of retinal veins. There are numerous exudates and intra retinal hemorrhage (Figure 1). Fluorescence angiogram revealed some areas of fluorescence leakage with areas of peripheral nonperfusion with hypoperfusion and some areas of neovascularization (Figure 2).
Ischaemic retinopathy was entertained and a collagen disease especially SLE was suspected.

She was advised for further investigations. Complete blood count (CBC) included hemoglobin at $7.9 \mathrm{~g} / \mathrm{dL}$ without hemolytic blood features, and normal white blood cell and platelet count. Erythrocyte sedimentation rate (ESR) was $28 \mathrm{~mm} /$ hour. Urine analysis found proteinuria and urinary total protein was $3.2 \mathrm{gm} /$ day. An antinuclear antibody (ANA) test was positive at a titer of 8.71 (positive $>1$ ) and anti-ds DNA > $240 \mathrm{IU} / \mathrm{ml}$ (positive $>25$ ). Her liver function tests were normal. She fulfilled five of the American College of Rheumatology criteria for SLE, Including arthralgia, photosensitivity, positive ANA, high titre of antidsDNA, and proteinuria.

The diagnosis of systemic lupus erythematosus was made. Anti-phospholipid antibody (APA) level was negative. Both Complements level were found low.
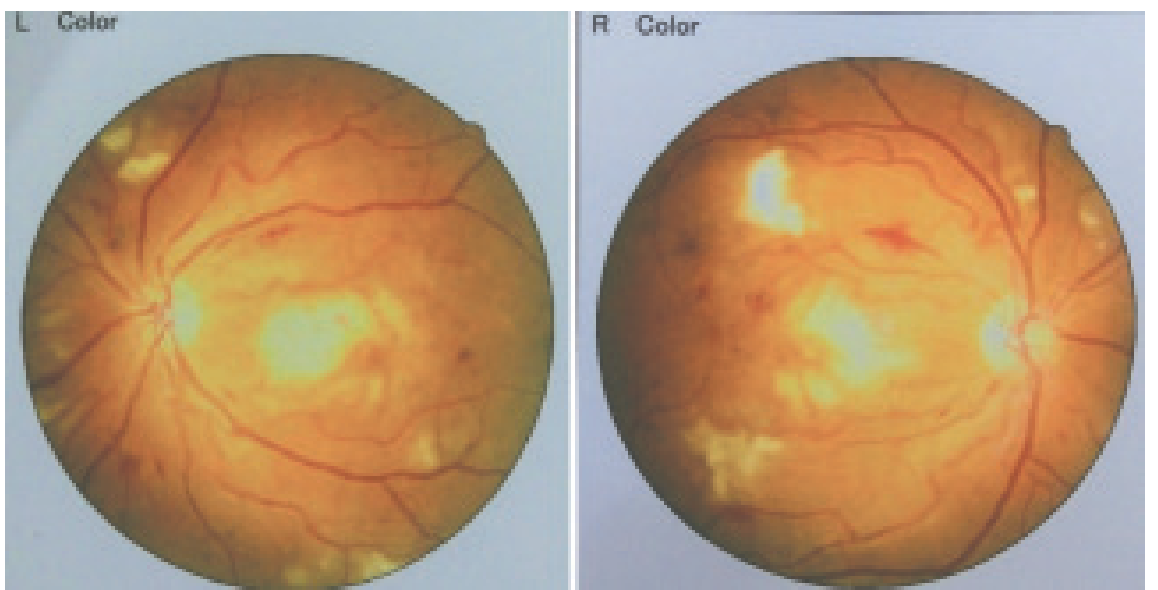

Fig.-1: Color fundus pictures at presentation showed dilated, tortuous and beading of retinal veins and numerous diffuse, confluent exudates and hemorrhage involving macula.
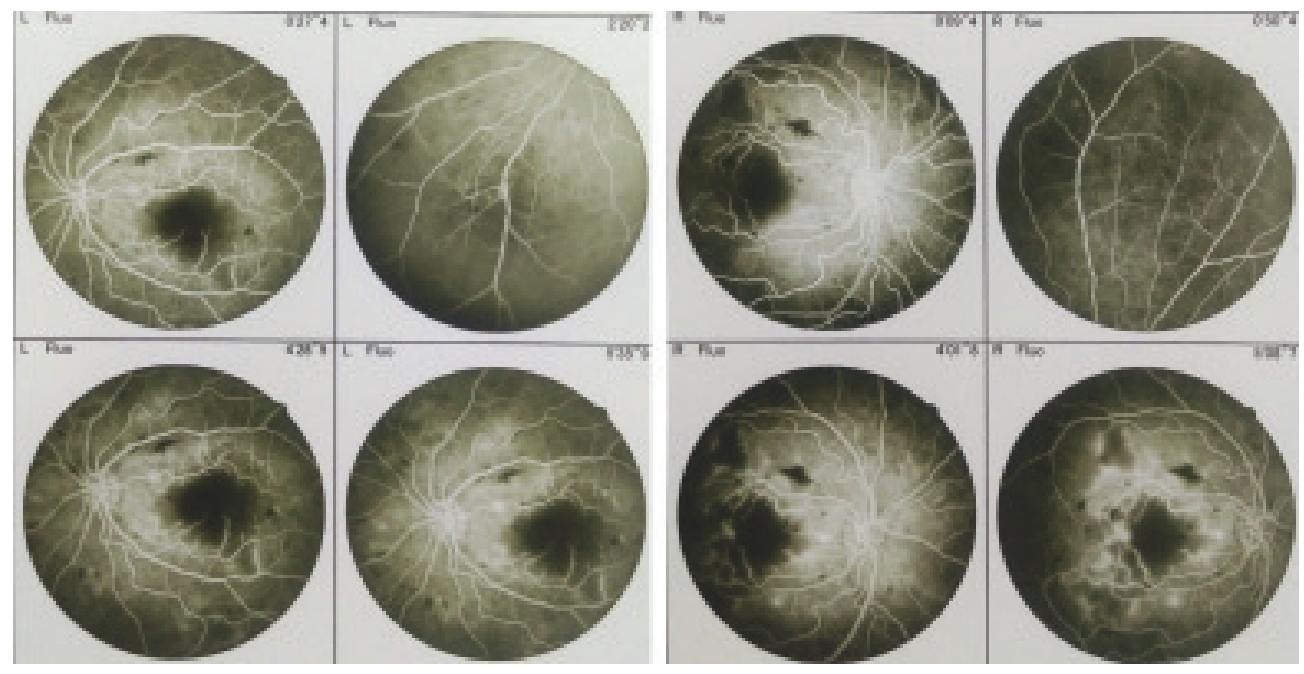

Fig.-2: Fluorescence angiography revealed areas of fluorescence leakage and peripheral nonperfusion with hypoperfusion and some areas of neovascularization. 


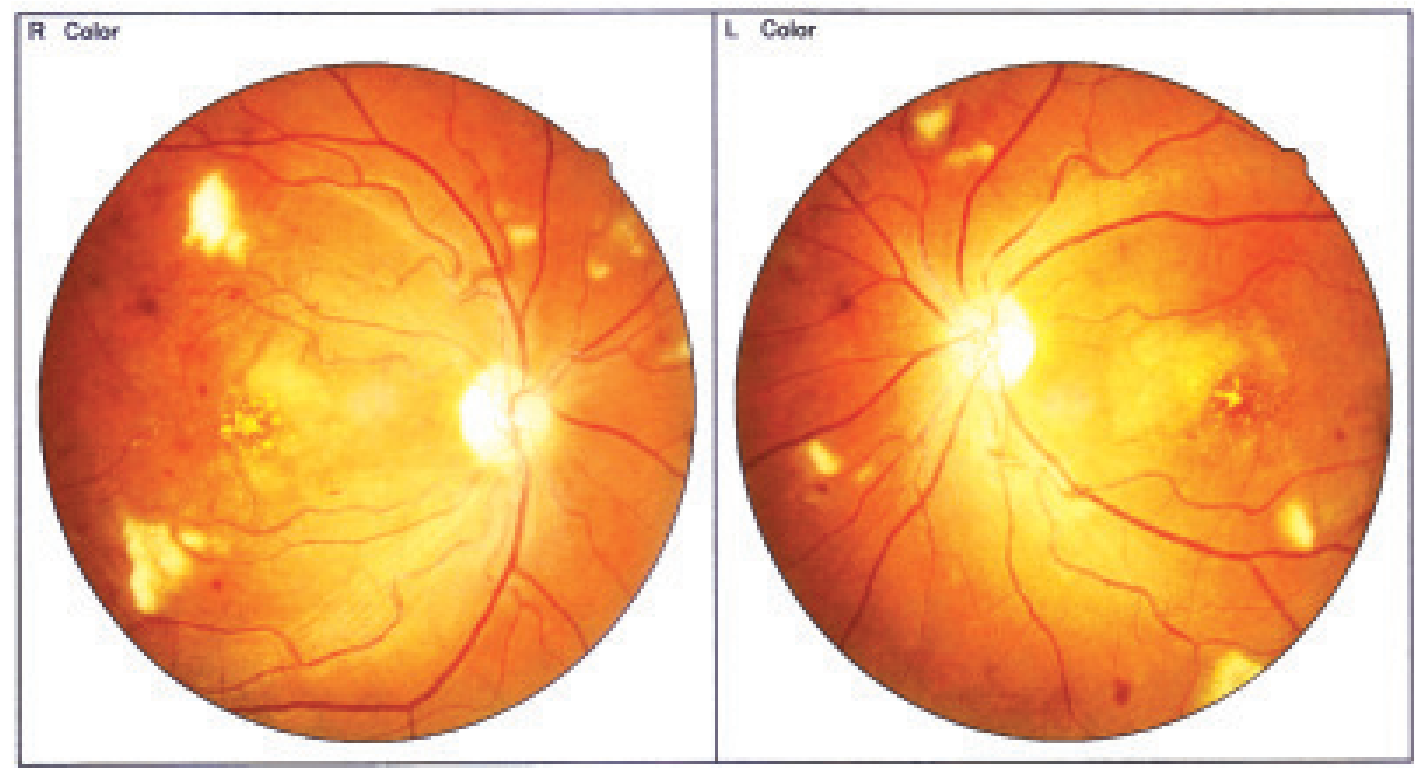

Fig-3: Four weeks after treatment with systemic steroid showed decreased exudates and haemorrhage

\section{Discussion}

SLE is a chronic, usually life-long, potentially fatal autoimmune disease characterized by unpredictable exacerbations and remissions with protean clinical manifestations. The etiology of SLE remains unknown. Disease manifestations result from recurrent vascular injury due to immune complex deposition, leukothrombosis, or thrombosis ${ }^{3}$.

Ocular findings in SLE include anterior segment signs such as keratoconjunctivitis sicca, episcleritis and scleritis. It is important to recognize SLE-associated posterior segment disease.Posterior segment manifestations of SLE include retinal microvasculopathy such as cotton-wool spots and retinal hemorrhages. Other manifestations may include microaneurysms, vascular tortuosity, arteriolar narrowing, retinal edema or exudates. Retinal microangiopathy associated with SLE is thought to represent immune complex-mediated vascular injury and microvascular thrombosis ${ }^{13}$.

Fortunately, most patients with mild retinopathy are at low risk for vision loss. ${ }^{14}$ In contrast, SLE associated vaso-occlusive disease characterized by widespread capillary non-perfusion of the retina has been associated with a worse visual prognosis. Up to 55 percent of patients may have vision worse than $20 / 200$, and 40 percent of patients may go on to develop retinal neovascularization and vitreous hemorrhage ${ }^{15}$. Patients often require panretinal photocoagulation to treat areas of capillary nonperfusion. Some patients can develop anterior segment rubeosis, tractional retinal detachment or macular infarction ${ }^{16}$ leading to significant vision loss and morbidity. Increasing evidence suggests that this more severe vaso-occlusive form of lupus-related retinopathy may be associated with the presence of antiphospholipid antibodies, including the presence of anticardiolipin (aCL) or lupus anti-coagulant antibodies (LAC) ${ }^{17}$.

Renal involvement is reported to occur in $61-81 \%$ of patients with SLE. A renal biopsy is necessary to confirm and classify the diagnosis of lupus nephritis and hence to guide treatment (Table 1).

Table-1

International Society of Nephrology/Renal pathologty society 2003 classification of $L N$

\begin{tabular}{ll}
\hline Class I & Minimal Mesangial LN \\
Class II & Mesangial proliferative LNLN \\
Class III & Focal LN (<50\% of glomeruli) \\
& III (A): acive lesions \\
& III (A/C): acive and chronic lesions \\
& III (C): chronic lesions \\
Class IV & Diffuse LN (50\% glomeruli) \\
& Diffuse segmental (IV-S) or global (IV-G) LN \\
& IV (A): acive lesions \\
& IV (A/C): acive and chronic lesions \\
& IV (C): chronic lesions \\
Class V & Membranous LN+ \\
Class VI & Advanced sclerosing LN (\%90\% globaly \\
& sclerosed glomeruli without residual \\
& activity) \\
\hline
\end{tabular}


Overall survival in patients with SLE is approximately $95 \%$ at 5 years after diagnosis and $92 \%$ at 10 years after diagnosis 18,19 . The presence of lupus nephritis (LN) significantly reduces survival to approximately $88 \%$ at 10 years. Our patient presented with features of stage IV (Diffuse proliferative lupus nephritis: DPLN) disease on renal biopsy. End stage renal disease or death has been reported in $20-50 \%$ of patients with this form of nephritis at 10-year followup due to progressive renal parenchymal injury ${ }^{20}$. Recommendations for management of LN consisted of pulse glucocorticoids followed by high-dose daily glucocorticoids in addition to an immunosuppressive medication, with cyclophosphamide (CYC) viewed as the most effective immunosuppressive medication for diffuse proliferative glomerulonephritis. Mycophenolate mofetil can be used as an alternative in child bearing aged patients.

Since the ocular complications of SLE are generally associated with active disease elsewhere in the body, control of the systemic disease may lead to resolution of the ocular manifestations. Some oral medications (e.g. nonsteroidal anti-inflammatory agents, antimalarial therapy, immunosuppressive agents, antiplatelet treatment, steroids) may be helpful in the treatment of $\mathrm{SLE}^{21}$. Systemic therapy with steroid is required when severe manifestations of SLE such as vaso-occlusive retinopathy are present ${ }^{21}$. In this case; intravenous steroid pulse therapy was prescribed in the beginning to stabilize the acute stage of the disease. Thereafter, antimalarial drugs, high-dose steroid and immunosuppressant were given to control the disease activity of SLE.

Treatment of SLE retinopathy is aimed at preventing complications arising from neovascularization. A significant factor affecting visual outcome is the high rate of neovascularization and vitreous hemorrhage arising from retinal ischemic events. Panretinal photocoagulation and vitrectomy have been found to be useful in preserving vision ${ }^{21}$. In this case, the early intervention with bilateral panretinal photocoagulation may be considered to have prevented complications arising from neovascularization.

Vision loss is an important issue in vaso-occlusive retinopathy of SLE. In 50\% of SLE patients with this type of vaso-occlusive retinopathy, visual acuity was less than 6/20 6-8. In our patient visual acuity of both eye improved to finger counting. The visual prognosis is poor, as reported in the literature ${ }^{6-8}$. This case highlights the importance of timely treatment to prevent further vision loss.

\section{Conclusion:}

This case report demonstrated that SLE might initially manifest with ocular symptoms, which are potentially severe. Though, Visual acuity improved after treatment, this case reminds us that in patients with severe vaso-occlusive retinopathy, a generalized immunologic disorder, like SLE, should be taken into consideration.

\section{References}

1. Rahman A, Isenberg DA. Systemic lupus erythematosus. N Engl J Med 2008;358(9):929-39.

2. Read RW. Clinical mini-review: systemic lupus erythematosus and the eye. Ocul Immunol Inflamm 2004;12(2):87-99.

3. Mills JA. Medical progress: systemic lupus erythematosus. N Engl J Med 1994;330:1871-9.

4. Au A, O’Day J. Review of severe vaso-occlusive retinopathy in systemic lupus erythematosus and the antiphospholipid syndrome: associations, visual outcomes, complications and treatment.Clin Experiment Ophthalmol 2004;32:87-100.

5. Ingram SB, Goodnight SH, Bennett RM. An unusual syndromeof a devastating non-inflammatory vasculopathy associated with antiphospholipid antibodies: report of 2 cases. Arthritis Rheum 1987;30:1167-71.

6. Montehermoso A, Cervera R, Font J, Ramos-Casals M,García-carrasco M, Formiga F, Callejas JL, et al. Association of antiphospholipid antibodies with retinal vascular disease in systemic lupus erythematosus. Semin Arthritis Rheum 1999;28:326-32.

7. Asherson RA, Merry P, Acheson JF, Harris EN, Hughes GRV.Antiphospholipid antibodies: a risk factor for occlusive vascular disease in systemic lupus erythematosus and the 'primary' antiphospholipid syndrome. Ann Rheum Dis 1989;48:358-61.

8. Fitzpatrick EP, Chesen N, Rahn E. The lupus anticoagulant and retinal vaso-occlusive disease. Ann Ophthalmol 1990;22:148-52.

9. Dooley MA, Aranow C, Ginzler EM. Review of ACR renal criteria in systemic lupus erythematosus. Lupus 2004;13:857-60.

10. Kasitanon N, Magder LS, Petri M. Predictors of survival in systemic lupus erythematosus. Medicine (Baltimore) 2006;85:147-56.

11. Ward MM, Pyun E, Studenski S. Mortality risks associated with specific clinical manifestations of systemic lupus erythematosus. Arch Intern Med 1996;156:1337-44.

12. Alarcon GS, McGwin G Jr, Petri M, Reveille JD, Ramsey-Goldman R, Kimberly RP. Baseline 
characteristics of a multiethnic lupus cohort: PROFILE. Lupus 2002;11:95-101.

13. Davies JB, Rao PK. Ocular manifestations of systemic lupus erythematosus. Curr Opin Ophthalmol 2008;19(6):512-8.

14. Klinkhoff AV, Beattie CW, Chalmers A. Retinopathy in systemic lupus erythematosus: Relationship to disease activity. Arthritis Rheum 1986;29(9):11526.

15. Jabs DA, Fine SL, Hochberg MC, et al. Severe retinal vaso-occlusive disease in systemic lupus erythematous. Arch Ophthalmol 1986;104(4):55863.

16. Shein J, Shukla D, Reddy S., et al. Macular Infarction as a Presenting Sign of Systemic Lupus Erythematosus. Retinal Cases \& Brief Reports 2008;2:55-60.

17. Au A, O'Day J. Review of severe vaso-occlusive retinopathy in systemic lupus erythematosus and the antiphospholipid syndrome: associations, visual outcomes, complications andtreatment. Clin Experiment Ophthalmol 2004;32(1):87-100.

18. Bernatsky S, Boivin JF, Joseph L, Manzi S, Ginzler E, Gladman DD, et al. Mortality in systemic lupus erythematosus. Arthritis Rheum 2006;54:2550-7.

19. Cervera R, Khamashta MA, Font J, Sebastiani GD, Gil A Lavilla P, et al. Morbidity and mortality in systemic lupus erythematosus during a 10-year period: a comparison of early and late manifestations in a cohort of 1,000 patients. Medicine(Baltimore) 2003;82:299-308.

20. Silverman ED.Pediatric systemic lupus erythematosus. In:Rudolh AM, Rudolph CD,Hostetter MK, Lister G,First L, Gershan A, editors. Rudolph pediatrics. $21^{\text {st }}$ ed. New York: McGraw-Hill: 2003.p.48-51

21. Peponis V, Kyttaris VC, Tyradellis C, Vergados I, Sitaras NM.Ocular manifestations of systemic lupus erythematosus: a clinical review. Lupus 2006;15:312 . 